\title{
Écologie et gestion forestière dans l'Indochine française
}

\author{
Par \\ FRÉDÉRIC THOMAS*
}

Les règlements et les aménagements forestiers en Indochine portent la trace de nombreuses problématiques de l'écologie. Cette science qui émerge à peine au début du siècle, a pénétré le milieu de la foresterie tropicale par divers canaux. Economie de la Nature, géobotanique, phytosociologie, écologie américaine donnent à la gestion forestière du colonisateur, une caution scientifique. De la mise en valeur, à la dépossession du droit d'usage de la forêt par les peuples qui l'habitent, en passant par la sauvegarde du milieu, l'écologie est une arme impérialiste subtilement malléable dès la période coloniale.

Mots-clés : Ecologie, colonialisme, économie de la nature, géobotanique, phytosociologie, écosystème, sylviculture tropicale.

The rules and adjustments of forest in Indochina bear the mark of numerous ecological issues. Ecology, hardly emerged at the dawn of century, has entered the field of tropical forestry through different ways. Economie de la Nature (according to C. von Linné), plantgeography, phytosociology and American ecology have given scientific securities to the forest administration. Be it used to dispossess the natives from their right to use the forest, or to protect environment, ecology was a subtly malleable weapon of imperialism during the colonization.

Key-words : Ecology, imperialism, nature policy, geography of plants, phytosociology, ecosystem, tropical forestry.

* Professeur d'histoire géographie, lycée de Longperrier

RFHM, t.85 (1998), n 319, p. 59 à 86. 
Pour comprendre convenablement un fait social, il faut l'appréhender totalement, c'est à dire $d u$ dehors comme une chose, mais comme une chose dont fait cependant partie intégrante l'appréhension subjective (consciente et inconsciente) que nous en prendrions si, inéluctablement homme, nous vivions le fait comme indigène au lieu de l'observer comme ethnographe. (Claude Lévi-Strauss, "Introduction à l'œuvre de Marcel Mauss» in Marcel Mauss, Sociologie et anthropologie. Quadrige, P.U.F. p. XXVII)

La colonisation française en Indochine, va devenir rapidement pour la foresterie française un lieu d'expérimentation et d'avancement de la connaissance botanique des milieux tropicaux. En retour le travail scientifique des forestiers servira le pouvoir colonial dans sa maîtrise de l'espace, des communications et des populations. Cette dynamique ne s'est bien sûr pas élaborée aussi facilement que sa formulation le laisse penser.

Le regard que les Européens ont porté sur ces forêts s'est lentement constitué. L'imaginaire de l'âge classique face aux mondes nouveaux n'autorisait qu'un regard ambivalent sur ces milieux fascinants et répulsifs. Face à la nature "sauvage ", l'Europe à la recherche d'un nouvel idéal social, projette des utopies transformistes, face à la nature "vierge" au contraire, elle s'éprend de primitivisme et cherche à préserver cet Eden ${ }^{1}$. Ainsi le milieu tropical humide existe avant tout dans la représentation que s'en donnent les Européens au travers les schèmes de leurs civilisations. Les forêts d'Indochine n'échappent pas à cette règle $^{2}$. Les explorateurs du XVIII ${ }^{\mathrm{e}}$ siècle dans leur grande majorité, imprégnés de la pensée des physiocrates et des premiers naturalistes, les perçoivent comme hostiles et monotones.

L'approche coloniale du $\mathrm{XIX}^{\mathrm{e}}$ siècle fixe de nouveaux regards. Les forêts d'Indochine deviennent des espaces à mettre en valeur. Dans une optique capitalistique, elles représentent une matière première à exploiter. La colonisation française, piquée de missions civilisatrices, d'œuvres scientifiques, et de bons sentiments, ne pouvait cependant se contenter d'un regard mercantiliste sur les

\footnotetext{
${ }^{1} \mathrm{C}$. Marouby, Utopie et primitivisme. Essai sur l'imaginaire anthropologique à l'âge classique. Travaux Seuil, 1990. $216 \mathrm{p}$.

${ }^{2}$ F. Thomas, La forêt tropicale humide vue dans les discours géographiques de la période coloniale. L'exemple de l'Indochine Française 1860 à 1943. Mémoire de Maîtrise, Université de Paris 1, 1995, 246 p.
} 
espaces forestiers. Les connaissances sur la forêt tropicale deviennent complexes et plurielles. Une approche globale de ce milieu émerge à la fin du XIX ${ }^{\mathrm{e}}$ siècle et au début $\mathrm{du} \mathrm{XX}^{\mathrm{e}}$. Cette approche globalisante est-elle à mettre en rapport avec l'émergence de l'écologie en Europe ?

\section{LA RÉGLEMENTATION FORESTIÈRE EN INDOCHINE FRANÇAISE}

"Les bois coloniaux d'Indochine" n'ont sans doute pas constitué une grande motivation dans la conquête de 1'Indochine par la France. F. Durand ${ }^{3}$ souligne toutefois que dès 1862 les amiraux généraux qui venaient de se mettre en place en Cochinchine, accordèrent des concessions forestières. Les premiers règlements forestiers furent pris à cette occasion pour organiser l'exploitation, les achats, le commerce, et visaient avant tout à pourvoir les nouveaux besoins en bois de la colonie, notamment pour la construction des premières lignes télégraphiques. Ils auguraient ainsi l'essentiel de la fonction de la réglementation forestière : assurer l'exploitation. A partir de 1879, les gouverneurs civils prennent le relais, et procèdent aux premières mises en réserve dans la province de Thudaumot. Jusqu'en 1888 l'exploitation demeure quasiment libre, date à laquelle un premier arrêté sur les autorisations de coupe est imposé au Tonkin qui vient à peine d'être pacifié. Il faut attendre 1901 pour que soit créé le premier service forestier d'Indochine. Très en retard en ce domaine par rapport aux Indes britanniques, les services forestiers français en Indochine vont cependant constituer un modèle pour l'ensemble des colonies françaises. Vont-ils alors constituer un contrepouvoir à la logique d'exploitation des forêts par l'administration générale ? Certains forestiers coloniaux à l'image de prédécesseurs en France, ont certainement cru pouvoir jouer la carte de la forêt avant celle des impératifs économiques et politiques ${ }^{4}$. Carte illusoire. Une tension apparaît effectivement dans le discours des administrateurs forestiers qui sont pris entre les impératifs d'exploitation et des velléités de sauvegarde de la forêt, mais les premiers l'emportent toujours. Les autorisations de coupes vont alors se généraliser pour

\footnotetext{
${ }^{3}$ F. Durand, Les forêts en Asie du Sud-Est. Recul et exploitation, le cas de l'Indonésie. L'Harmattan, 1994, 411p.

${ }^{4}$ Voir à ce propos Les Eaux et Forêts du 12è au 20è siècles. Sous la dir. de L. Bardé, Centre National de la Recherche scientifique, 1987. p.484 à 490. Les auteurs, issus des Eaux et Forêts euxmêmes, montrent comment l'Ecole royale forestière de Nancy à son ouverture a été vécue par la direction générale des Eaux et Forêts comme une force d'opposition à la logique commerciale des maîtres de forge dans l'exploitation des forêts françaises. L'opposition entre Legrand, directeur général des forêts et Lorentz directeur de l'école de Nancy donne le ton à des forestiers qui se plairont à se considérer frondeurs. Cette attitude relève, cependant plus, particulièrement sur le terrain colonial, de l'auto-proclamation que de la réalité, les intérêts individuels dans ce type d'administration l'emportant généralement sur les sacrifices aux grandes causes.
} 
assurer une production rationnelle, fournir ses subsides à l'administration forestière et satisfaire, accessoirement, les exigences de conservation du patrimoine $^{5}$. En 1930, une refonte des législations entraine une plus ou moins grande harmonisation pour tout le domaine forestier d'Indochine. Mais bien avant cette date, on peut le subdiviser en deux entités clairement individualisées : le domaine forestier classé, le domaine forestier protégé ${ }^{6}$.

Le domaine protégé, de loin le plus vaste, est le lieu de production. Sans entrer dans le débat sur la mesure et les critères de mesure des surfaces forestières en Indochine dans la période coloniale ${ }^{7}$, on peut considérer qu'il constitue $90 \%$ de la surface boisée. Contrairement à son nom, il est ouvert à l'exploitation mais fait l'objet de restrictions, permettant de ne pas parler d'exploitation libre.

Le domaine forestier protégé (autrefois dénommé forêts libres) est de beaucoup le plus vaste et fournit, comme nous le verrons plus loin, la grande majorité des bois, produits secondaires et sous-produits livrés au commerce. Dans les forêts de ce domaine, on laisse à l'exploitation le caractère de cueillette qu'elle affectait depuis toujours en Indochine, en la soumettant cependant aux quelques règles suivantes susceptibles à la fois de sauvegarder les intérêts financiers de la Colonie et, autant que possible, de faire durer les peuplements. Tout exploitant doit se munir d'un permis d'exploitation l'autorisant à extraire un certain nombre de pièces de bois ou une quantité d'autres produits forestiers dans un massif déterminé et dans un délai fixé. Il est, en outre, astreint à observer certaines règles : coupe rez-terre, recépage des sujets cassés par l'abattage et surtout, dans le but d'éviter la ruine des forêts ainsi soumises à la coupe libre, aucun arbre ne peut être abattu s'il n'a pas au moins certaines dimensions dites "diamètre minima d'abattage". Cette mesure conservatrice, si elle ne peut empêcher la ruine de la forêt, en recule cependant l'échéance ; elle était indispensable pour empêcher la disparition dans un court délai, des tiges de petites dimensions des meilleures essences, celles auxquelles les bûcherons s'attaquaient de préférence, notamment pour la fourniture de bois de chauffage et parce que l'abattage en était facile. (P. Maurand 1943) ${ }^{8}$

\footnotetext{
${ }^{5}$ Dès 1908 C. Guyot, directeur de l'école de Nancy, dans son cours de droit forestier développe l'idée que la forêt est un patrimoine commun.

${ }^{6}$ Le domaine communal et les forêts privées ne constituant pas des surfaces importantes ne seront pas traitées dans cet article.

${ }^{7}$ F. Durand, op. cit., p. 17, 42-43, et surtout p.132-174. F. Durand, dans ces pages, compare les chiffres de différentes organisations nationales et internationales des superficies boisées en Asie du Sud-Est, il montre aisément comment les critères retenus dans ces statistiques peuvent faire varier les statistiques de façon conséquente à des fins idéologiques.

${ }^{8} \mathrm{P}$. Maurand, L'Indochine forestière : les forêts d'Indochine, exploitation; défrichement, aménagement, reconstitution des forêts. Imprimeries d'Extrême Orient, 1943, p. 22.
} 
Les restrictions imposées par le service forestier ne sont en fait que des contraintes d'exploitation. Le tableau suivant, exprimé en piastres, tiré d'un ouvrage de A. Bertin, les synthétise ${ }^{9}$.

\begin{tabular}{|c|c|c|c|c|}
\hline & Tonkin & Cambodge & Cochinchine & Annam \\
\hline \multicolumn{5}{|l|}{ Bois d'oeuvre } \\
\hline \multicolumn{5}{|l|}{ Prix du permis } \\
\hline a) Essences classées & $0,30 \$ / 5$ pièces & $1,50 \$ / 5$ pièces & $1,50 \$ / 5$ pièces & $2 \$ / 10$ pièces \\
\hline b) Essences non classées & $0,15 \$ / 10$ pièces & $1,50 \$ / 5$ pièces & $1,50 \$ / 25$ pièces & $2 \$ / 30$ pièces \\
\hline \multicolumn{5}{|l|}{$\begin{array}{l}\text { Redevance à titre de prix } \\
\text { de vente par } \mathrm{m}^{3}\end{array}$} \\
\hline Bois de cercueil & $10,0 \%$ & & & \\
\hline Bois de luxe en grume $(G)$ & $4,8 \%$ & & $4,8 \%$ & $4,0 \%$ \\
\hline Bois de luxe équarris $(\mathrm{E})$ & $6,8 \%$ & & $6,8 \%$ & $6,0 \%$ \\
\hline $1^{\text {ère }}$ catégorie $(\mathrm{G})$ & $3,5 \%$ & $3,0 \%$ & $3,5 \%$ & $2,5 \%$ \\
\hline $1^{\text {ère }}$ catégorie $(E)$ & $4,8 \%$ & $4,3 \%$ & $4,8 \%$ & $3,8 \%$ \\
\hline $2^{\text {ème }}$ catégorie $(\mathrm{G})$ & $2,0 \%$ & $1,8 \%$ & $1,8 \%$ & $1,5 \%$ \\
\hline $2^{\text {ème }}$ catégorie $(\mathrm{E})$ & $2,5 \%$ & $2,5 \%$ & $2,5 \%$ & $2,2 \%$ \\
\hline $3^{\text {ème }}$ catégorie $(\mathrm{G})$ & $1,0 \%$ & $0,8 \%$ & $1,0 \%$ & $0,7 \%$ \\
\hline $3^{\text {ème }}$ catégorie $(\mathrm{E})$ & $1,5 \%$ & $1,1 \%$ & $1,3 \%$ & $1,0 \%$ \\
\hline $4^{\text {ème }}$ catégorie $(\mathrm{G})$ & $0,6 \%$ & $0,5 \%$ & $0,6 \%$ & $0,4 \%$ \\
\hline $4^{\text {ème }}$ catégorie $(\mathrm{E})$ & $0,8 \%$ & $0,7 \%$ & $0,8 \%$ & $0,6 \%$ \\
\hline \multicolumn{5}{|l|}{ Bois de feu } \\
\hline $\begin{array}{l}\text { Permis } \\
\text { Redevance }\end{array}$ & $\begin{array}{l}10 \$ / 10 \text { Comm. } \\
0,15 \$ / \text { Stère }\end{array}$ & $\begin{array}{l}6 \$ / 20 \text { Comm. } \\
0,06 \$ \text { / Stère }\end{array}$ & $\begin{array}{c}10 \$ / 400 \text { Stères } \\
0,2 \$ / \text { Stère }\end{array}$ & $\begin{array}{c}5 \$ / \text { an } \\
0,15 \$ / \text { Stère }\end{array}$ \\
\hline & & & & \\
\hline
\end{tabular}

Tiré de A. Bertin, 1924

Comme on peut l'observer, le régime des taxes n'est pas le même dans toutes les colonies d'Indochine et selon la catégorie de bois exploité. Ces différences révèlent les difficultés du service forestier à satisfaire les intérêts marchands à court terme et la recherche d'une exploitation non prédatrice. Loin de préoccupations écologiques telles qu'on les entend à la fin du $\mathrm{XX}^{\mathrm{e}}$ siècle, cette tarification vise à limiter l'exploitation de bois de feu (peu capitalistique) en imposant de lourds permis, particulièrement dans les régions densément peuplées; et à optimiser celle d'essences classées, peu taxées dans les régions d'accès difficile. Elle est, comme le reconnaît $P$. Maurand dans les années quarante, "fixées pour encourager l'exploitation sous le contrôle de l'administration ».

9 A. Bertin, Régimes forestiers dans les colonies françaises. Paris, La vie technique et industrielle, 1924, p.60. 
Dans le domaine réservé, la forêt se régénère, c'est le lieu expérimental du service forestier. Aménagements, enrichissements, plantations, arboretum ont pour finalité d'assurer une "exploitation méthodique", c'est-à-dire une exploitation qui prélève seulement dans la forêt la quantité de bois que celle-ci est capable dans le même temps de reconstituer. Ce concept-clef dans le discours des forestiers permet de montrer une colonisation non prédatrice qui prend soin de ne pas épuiser le milieu qu'elle exploite. La colonisation ne détruit pas, elle met en valeur. Cependant la faiblesse des mises en réserve est évidente. En 1928, selon le Congrès forestier qui se tînt à Grenoble ${ }^{10}$, seulement $6,8 \%$ du domaine forestier en Indochine est mis en réserve. Cette proportion augmente sensiblement au cours de la période coloniale, mais ne dépassera jamais les $10 \%{ }^{11}$. De plus, sur cet espace, les travaux d'aménagement sont extrêmement restreints. En 1943, si l'on suit les statistiques de P. Maurand seulement 573.470 ha sont aménagés, c'est-à-dire $17 \%$ du domaine réservé, soit $2 \%$ de la surface forestière totale. La production de bois assurée par les réserves aménagées ne vaut guère mieux comme, le montre le tableau suivant.

\begin{tabular}{|l|c|c|c|c|}
\hline & Annam & Tonkin & Cochinchine & Cambodge \\
\hline Bois d'œuvre & & & & \\
\% coupe méthodique & 2,3 & 0,3 & 6,2 & 1,2 \\
\% coupe libre & 97,7 & 99,7 & 93,8 & 98,8 \\
\hline Bois de feu & & & & \\
\%coupe méthodique & 6,4 & 25,4 & 45,0 & 20,5 \\
\% coupe libre & 93,6 & 74,6 & 55,0 & 79,5 \\
\hline
\end{tabular}

Tiré de P. Maurand, 1943

De l'aveu même de P. Maurand, en 1943, la production de bois en exploitation méthodique est quasiment nulle.

Ce sont donc les forêts protégées qui devront encore assurer toute la production de bois d'œuvre, en attendant que les réserves soient en état, après reconstitution de leur peuplement, d'être aménagées et fournir d'une façon continue et constante ce bois

\footnotetext{
${ }^{10}$ Les ressources forestières d'Indochine. Rapport présenté au Congrès Forestier International de Grenoble en 1925 par le Gouvernement Général d'Indochine. p. 5.

${ }^{11}$ Calculé par nous, d'après P. Maurand, L'Indochine forestière : les forêts d'Indochine, exploitation, défrichement, aménagement, reconstitution des forêts. op. cit. p. 26.
} 
d'œuvre nécessaire au pays. Mais se sera très long et les forêts libres ne seront-elles pas ruinées auparavant? »(P. Maurand, 1943) ${ }^{12}$

Les statistiques montrent très clairement que l'objectif d'exploitation non destructrice était hors de portée. L'alibi de "l'exploitation méthodique" ne résiste pas à l'examen et la logique de la mise en réserve n'est donc pas la protection des forêts, mais la constitution d'un capital forestier exploitable à moyen terme. Pour ne pas gêner l'exploitation du présent, les forêts mises en réserve n'étaient pas les plus riches mais au contraire celles totalement appauvries par l'exploitation. Le service forestier devait tant bien que mal les régénérer en 60 ou 80 ans.

L'administration agissant avec une sage modération, les mises en réserves ne se firent et continuent à ne se faire que progressivement et sans rien brusquer. Les forêts ainsi mises en réserve sont le plus souvent des forêts où les exploitations libres ne trouvent pour ainsi dire plus rien de bon à enlever comme bois d'œuvre. (H. Guibier, 1926) ${ }^{13}$

Une fois la forêt reconstituée, l'abandon en concession à des compagnies privées constituait le couronnement des investissements du service forestier.

Un cahier des charges, annexé à l'arrêté, fixe les conditions d'exploitation. Des contrats spéciaux à long terme, durée maxima 30 ans, peuvent être accordés pour favoriser les entreprises industrielles commerciales qui trouveront dans les réserves aménagées un aliment régénéré indéfiniment pour une industrie de grande durée : scierie, pâte à papier, distillation du bois, alcool à bois. Les capitalistes de la métropole commencent à chercher ces contrats qui consacrent l'aménagement des réserves forestières. (A. Bertin, 1924) ${ }^{14}$

Le cahier des charges des contrats à long terme, figurant dans le procès verbal d'adjudication, n'était pas plus contraignant que les règles de sylvicuture imposées sur le domaine protégé, tout au plus le service forestier pouvait exercer un meilleur contrôle sur l'exploitant ${ }^{15}$. La rareté des grandes compagnies forestières, en faisait des clients à qui l'on doit des égards, et dans ces conditions le service forestier, ne pouvait exercer des contraintes pesantes dans le cahier des charges $^{16}$. On peut mesurer, au détour de quelques remarques, le souci de

${ }^{12}$ P. Maurand, L'Indochine forestière...op. cit., p. 26.

13 H. Guibier, "Caractères généraux de la forêt d'Indochine». in H. Lecomte, Les bois d'Indochine. Gouvernement Général d'Indochine. p. 280.

${ }^{14}$ A. Bertin, Régimes forestiers dans les colonies françaises. op. cit., p. 24.

${ }^{15}$ Archives Nationales du Vietnam (Hanoi), Dir. de l'Agriculture, des forêts et du Commerce, Série N 9, Dossier $n^{\circ} 623$.

${ }^{16}$ Le nombre de compagnies forestières était extrêmement limité. La société des allumettes du Tonkin, les Papeteries, l'East Asiatic et la Bien Hoa Forestière sont à peu près les seules grandes entreprises, l'essentiel de l'exploitation étant assurée par un ancien et dynamique réseau de marchands chinois. Les adjudications de coupe sur le domaine protégé se font au prix d'annonce, les enchères ne montent jamais, à tel point que certaines adjudications se font par vente au rabais. Archives nationales du Vietnam (Hanoi), Dir. de l'Agriculture des forêts et du Commerce, Série N 9, Dossiers 639 à 642. 
l'administration d'attirer les capitaux de ces grosses compagnies. A. Bertin, à propos des grandes exploitations en Annam, nous révèle quelques-unes de ces facilités : "Lorsqu'une surface boisée intéressera une concession minière, les bois seront abandonnés gratuitement en cas d'utilisation sur place pour la mine. » (A. Bertin, 1924) ${ }^{17}$

$\mathrm{Au}$ total, la réglementation forestière répond d'abord à cette formule lapidaire : assurer la production de demain sans gêner celle d'aujourd'hui. Mais le colonialisme exige aussi d'elle, afin de masquer cette logique, de démontrer l'impérieuse nécessité de l'administration moderne. La rhétorique la plus pertinente consiste à montrer que le service forestier existe pour concilier les intérêts divergents de l'exploitant, de l'indigène et de la forêt elle-même. La petite philosophie républicaine y retrouvait les siens : intérêt général, contrat social, etc.

Il faut donner au concessionnaire la stabilité de la durée, et il sera ainsi intéressé lui-même à améliorer les peuplements forestiers. (...) L'exploitant est un auxiliaire indispensable de la mise en valeur des massifs. Il faut donc l'attirer et lui permettre de prospérer et de s'enrichir. Mais sa prospérité est liée à l'amélioration de la forêt que cherchent également les services forestiers. Tout le secret d'un bon régime réside dans la parfaite entente entre le forestier et l'exploitant, l'un ne pouvant se passer de l'autre, dans le présent et dans l'avenir. (A. Bertin, 1924) ${ }^{18}$

Cette conception du rôle du service forestier, cramponnée aux mythes fondateurs de la République et de la colonisation, assurant de surcroît l'exploitation commerciale explique l'articulation domaine protégé / domaine réservé, l'un dégageant des profits investis dans le second. L'administration forestière procédait ainsi à un superbe renversement de logique, la colonisation sauvait la forêt. L'exploitation en générant des capitaux par la mise en valeur, devenait la condition de la conservation de la forêt, sublime paradoxe qui effaçait le fait qu'elle la détruise. La philosophie républicaine et le paternalisme colonial se prêtaient cependant mal, par la généralité et l'étendu de leurs objets, à la foresterie coloniale. Il fallait trouver d'autres justifications plus proches de la sylviculture pour voler au secours de l'action des forestiers. Comment l'écologie qui n'apparaît dans sa formulation contemporaine que dans l'entre-deux-guerres et plus particulièrement dans les pays anglo-saxons, a-t-elle pu jouer ce rôle ? Pour dépasser rapidement cette aporie, avant de préciser notre pensée, nous nous appuierons sur C. Elton qui en 1927, constatait : "L'écologie : un nouveau nom

\footnotetext{
${ }^{17}$ ibid., p. 24.

${ }^{18}$ ibid., p. 43.
} 

pour un très vieux sujet. Cela signifie simplement histoire naturelle
scientifique. ${ }^{19}$.

\section{MISE EN VALEUR ET POLICE DE LA NATURE}

Les services forestiers en Indochine vont disposer d'un terrain privilégié pour multiplier les expérimentations en matière de sylviculture tropicale. C'est peutêtre, comme nous allons le voir, par ces travaux d'aménagement que l'écologie pénètre la sylviculture. Mais il est difficile de faire la mesure entre la réflexion théorique venue des différentes écoles d'écologie et la part des observations empiriques. Sans doute des interactions nombreuses existèrent entre ces savoirs pratiques et les savoirs scientifiques des universités et des laboratoires en Europe. Ces travaux, au début de la colonisation, surprennent par leur radicalisme. En effet jusqu'au milieu des années vingt ils ont pour ambition, afin de satisfaire les demandes du marché, une totale recomposition de l'anarchie végétale que représente les forêts tropicales. Les années trente font place à plus de pragmatisme, tant face au marché, qu'aux contraintes des écosystèmes. Les forestiers révisent leur projet pour intégrer les données de l'environnement.

Les forêts d'Indochine déconcertent avant tout le forestier qui arrive des zones tempérées par leur richesse floristique, qui est aussi la cause de leur pauvreté commerciale. "Elle n'est pas d'une grande richesse en bois utilisable, ne permettant d'extraire que $100 \mathrm{~m}^{3} / \mathrm{ha}$. soit l'équivalant de 1 à 2 arbres. » (A. Chevalier, 1946) ${ }^{20}$

Cette estimation paraît même aux yeux de beaucoup très optimiste ; on calcule plus souvent qu'une forêt tropicale ne possède en moyenne que 10 à 20 $\mathrm{m}^{3}$ de bois exploitable à l'hectare, contre 200 pour les forêts tempérées. En Indochine par exemple on ne trouve qu'un teck pour trois hectares, nous dit P. Deffontaines $^{21}$. Les observations des forestiers et les problèmes d'exploitation font souhaiter une recomposition des forêts d'Indochine vers le paradigme productiviste de la grande futaie. L'horizon limité des représentations de la forêt par les écoles forestières tant allemandes que françaises, balançant sans nuance du taillis à la futaie, avec pour seule forme intermédiaire le taillis sous-futaie,

\footnotetext{
${ }^{19}$ C. Elton, Animal Ecology. Londres, Mathuen \& Science Paperbacks, 1971, éd. originale 1927, p. 1. Cité par J.-Marc Drouin, L'écologie et son histoire. Réinventer la nature. Champs Flammarion, 1991, p. 34.

${ }^{20}$ A. Chevalier \& D. Normand, Forêts vierges et bois coloniaux. Paris PUF, 1946, p. 23.

${ }^{21}$ P. Deffontaines, L'homme et la forêt. Paris NRF, 1933, p. 151.
} 
pouvait d'autre part n'accoucher que d'un projet de "peuplement pur"22. H. Lecomte, tout imprégné de cette sylviculture, voit l'exploitation forestière tropicale comme une culture de plantation.

De même que le caféier, le cacaoyer, le cotonnier, etc., doivent faire l'objet d'une culture suivie pour que la production devienne régulière, de même aussi la production de bois ne peut être livrée au hasard et les forêts hétérogènes actuelles, au fur et à mesure de leur exploitation, doivent être remplacées par des forêts aussi homogènes que possible et aménagées pour une exploitation ultérieure. Quelques mesures ont déjà été prises dans ce sens ; mais il importe que les prescriptions soient rigoureusement suivies. Il est désirable en effet que nos successeurs soient en mesure de trouver dans nos colonies les différents bois qui peuvent leur être utiles et que cette exploitation puisse se poursuivre dans les mêmes conditions que celle des forêts de la métropole. Pour obtenir un tel résultat, il est nécessaire d'appliquer aux forêts coloniales les règles ordinaires de la sylviculture et par conséquent la création d'un corps spécial d'agents forestiers coloniaux paraît indispensable. (H. Lecomte, 1923) ${ }^{23}$

Il s'agit donc d'homogénéiser la forêt dense humide autant que la technologie et les moyens peuvent le permettre. Le botaniste Lecomte se fixe l'objectif d'une recomposition lente des forêts au fur et à mesure de leur exploitation, mais qui vise à long terme à une transformation radicale du milieu ; une transmutation, basée sur un triptyque chimérique. Le premier pan de l'utopie consiste à considérer la forêt comme transformable selon les règles de la sylviculture européenne. La croyance que l'on peut la faire évoluer vers des hautes futaies de peuplement pur n'est pas mise en doute en 1923. L'utopie réside aussi, comme on l'a vu dans la réglementation forestière, dans l'espoir de la mise en place de l'exploitation méthodique qui permettrait d'éviter l'épuisement des forêts. Enfin, dernier panneau du triptyque de la transmutation de la forêt dense humide chez Lecomte, l'exploitation durable (pour prendre un terme plus contemporain) est faite pour les générations coloniales de demain, le cadre colonial étant perçu comme permanent (le contraire eut été difficilement imaginable, le colonisateur n'investissant pas pour des générations décolonisées) : "L'avenir est donc aux forêts aussi homogènes que possible, aménagées suivant les règles de la sylviculture, en vue des exploitations de l'avenir. » (H. Lecomte, 1926) ${ }^{24}$.

En quoi peut-on rattacher cette volonté d'asservissement de la nature à l'écologie? Pour répondre à cette question, un détour par les paradoxes de la pensée naturaliste du XVIII ${ }^{\mathrm{e}}$ siècle est très éclairant. Pareille à la modernité qui

\footnotetext{
${ }^{22}$ A propos des limites des représentations forestières voir J.-J. Dubois, Espaces et milieux forestiers dans le nord de la France. Etude de biogéographie historique. Thèse de doctorat d'Etat ès Lettres et Sciences Humaines, 1989, 843 p.

${ }^{23}$ H. Lecomte, Les bois coloniaux, Paris A. Colin, 1923, p. 181.

${ }^{24} \mathrm{H}$. Lecomte, Les bois d'Indochine. op. cit., p. 1.
} 
sépare sujet/objet, le naturalisme occidental opère une fracture entre nature et culture. Cette objectivation de la nature permet un bond dans la connaissance des espèces animales et végétales notamment grâce à leur recensement systématique et à une classification rationnelle. Mais cette objectivation de la nature ne conduit pas à son désenchantement, paradoxe d'une pensée objectivante qui refuse encore tout matérialisme et conserve l'approche d'un Tout sacré ${ }^{25}$. C von Linné illustre parfaitement ce traitement ambivalent de la nature. Son travail est le fruit d'une grande attention pour le plus infime détail (matériel) de la nature qui loin de la séculariser, débouche en dernier ressort sur un équilibre qui ne peut être que l'œuvre de Dieu : "Par économie de la nature on entend la très sage disposition des êtres naturels instaurée par le Souverain Créateur, selon laquelle ceux-ci tendent à des fins communes et ont des fonctions réciproques » (C. Von Linné entre, 1749 et 1769$)^{26}$.

Cette perception de l'organisation de la nature, ou chaque élément tient une place et un rôle particulier, préfigure, dans une version sacrée, la notion de climax biotique et fait de l'économie de la nature, une préécologie ${ }^{27}$; préécologie ou proto-écologie qui n'interdit cependant pas une modification de la nature par l'homme, puisque centre de la Création, elle lui est assujettie. Ainsi elle devient une science de l'organisation scientifique de la nature en vue de sa "mise en valeur" pour l'humanité. Pour Buffon et les physiocrates, la nature sauvage est laide car improductive donc inamicale, il faut la réorganiser. La transformation des forêts d'Indochine trouvera sa raison scientifique dans cette écologie orthopédique des premiers naturalistes. C'est au nom d'un équilibre tourné vers l'homme que les forestiers en bonne conscience formulent un véritable projet écocide. Y. Marcon, conservateur des forêts en Indochine, définit d'ailleurs sans ambages le rôle qu'il dévolue au service forestier.

Dans ce cadre nous laisserons de côté tout ce qui se rapporte à la protection de la flore naturelle, activité très intéressante, certes, mais qui s'attache plutôt à des considérations d'ordre sentimental ou de science pure, pour ne nous occuper que de l'activité économique du Service Forestier. (...) Le Service Forestier est un organisme administratif destiné à conserver ou créer les boisements nécessaires d'un pays et à les traiter de manière à en tirer la plus grande somme de produits ou d'effets utiles. (Y. Marcon, 1938) ${ }^{28}$

${ }^{25}$ A. Koyré, Du monde clos à l'univers infini. (1957) Tel Gallimard, 1973, 349 p.

${ }^{26}$ C. Von Linné, L'équilibre de la nature. Introduction et notes de C. Limoges, Paris, Vrin, 1972. Cité par J.-P. Deléage, Une histoire de l'écologie. Paris, La Découverte, 1991, p. 29.

${ }^{27}$ Nous empruntons cette thèse aux auteurs suivants : P. Acot, Histoire de l'écologie. Paris, PUF, 1994, 127 p. J.-P. Deléage, Une histoire de l'écologie. op.cit.,330 p. J.-Marc Drouin, L'écologie et son histoire. Réinventer la nature. Champs Flammarion, 1991, 213 p.

${ }^{28} \mathrm{Y}$ Marcon, Le rôle des services forestiers aux colonies. Actes de comptes-rendus de l'Association colonies sciences, 1938, p. 2. 
L'écologie s'entend, ici, non pas dans un sens de protection de la flore, préoccupation reléguée au rang de romantisme chez cet auteur mais de maintien d'un certain équilibre du biotope pour une utilisation optimale des produits forestiers. Loin de tout préservationnisme (et même de conservationnisme), le service forestier s'appuie bien néanmoins sur des notions d'équilibre global, fondement de l'écologie moderne.

\section{AMÉNAGEMENTS FORESTIERS ET ÉCOLOGIE SCIENTIFIQUE}

Très vite cependant, les projets transformistes de la forêt qui en résultent, apparaissent irréalistes. Ce sont les phytosociologues, qui sur le milieu tropical, en prennent d'abord la mesure En effet, du point de vue de la phytosociologie, l'analyse spatiale de la répartition des espèces se heurte, non pas à la richesse floristique mais à son corollaire, des lois de distribution incompréhensibles. Pour les saisir, les forestiers empruntent à deux concepts qui s'opposent au début du $\mathrm{XX}^{\mathrm{e}}$ siècle, "la formation" et "l'association" végétale. Le premier, le plus ancien, aboutissement de la pensée humboldtienne, est une approche macroscopique de la végétation. Il s'intéresse à la corrélation entre la physionomie du groupement végétal et les contraintes exogènes (sols, climats, reliefs...). Dans la pensée évolutionniste de la période, ses tenants sont plutôt lamarckiens car ils conditionnent l'évolution des formes vivantes aux contraintes environnementales. La formation trouve son plein développement avec E. Warming et W. P. Shimper qui sortent la biogéographie de son travail chorographique pour fonder une géobotanique écologique. L'écologie, grâce à ces auteurs, acquiert pour la première fois un statut scientifique : méthode d'analyse dont l'objet sont les formations végétales.

L'écologie cherche :

$\left.1^{\circ}\right)$ à déterminer quelles sont les espèces qui se retrouvent généralement associées dans les mêmes habitats (stations) (...).

$2^{\circ}$ ) à tracer la physionomie de la végétation et du paysage (...).

$3^{\circ}$ ) à répondre aux questions suivantes :

- pourquoi chaque espèce a-t-elle un comportement et un habitat qui lui sont propres?

- pourquoi les espèces se groupent-elles en communautés ?

- pourquoi celles-ci ont-elles une physionomie caractéristique ?

$4^{\circ}$ ) à étudier les problèmes concernant l'économie des plantes, leur exigence vis-à-vis du milieu et les moyens qu'elles emploient pour tirer parti des conditions environnantes et adapter à cette fin leur structure interne et externe et leur forme générale. (E. Warming $1895)^{29}$

\footnotetext{
${ }^{29}$ E. Warming, Oecology of plants, Oxford, 1909, p. 2. Cité par J.-M. Drouin, op. cit., p. 73.
} 
L'écologie qui était jusqu'ici une façon de penser la géographie des plantes devient le fondement de celle-ci. Les historiens de l'écologie voient dans Warming et Shimper la naissance de l'écologie scientifique.

Le second concept, l'association, émerge seulement en 1913, autour de J. Braun-Blanquet. C'est une critique du premier, jugé flou et imprécis du point de vue taxinomique. L'association peut donc par opposition être qualifiée de mésoscopique et, plus darwinienne, elle délaisse les facteurs exogènes, pour se concentrer sur les relations entre espèces du groupement (en lutte pour la vie). En France, pendant toute la première moitié $\mathrm{du} \mathrm{XX}^{\mathrm{e}}$ siècle, l'école de Phytosociologie de Zurich-Montpellier autour de Braun-Blanquet est assez dominante et impose l'association à tout travail de phytosociologie. Certains auteurs y voient aujourd'hui un frein à la pénétration en France des idées de l'écologie qui fleurissent à la même époque dans les pays anglo-saxons ${ }^{30}$.

Où se situent les forestiers dans ce débat? Le cours de géographie botanique que P. Guinier dispensait à l'école des Eaux et Forêts à Nancy en 1932 est à ce titre tout à fait intéressant ${ }^{31}$. "L'étude des associations prime aujourd'hui l'étude des formations » précise Guinier, confirmant ce constat de domination de l'école zuricho-montpellièraine $^{32}$. Mais loin de s'en contenter, il recommande aux forestiers un travail de phytosociologie en deux temps. Le premier est une approche qu'il qualifie "d'écologique» pour déterminer la «formation». Cette "notion instinctive ${ }^{33}$ doit être complétée par une analyse floristique plus précise, deuxième temps du travail du phytosociologue. Dans la querelle Association / Formation, les forestiers de Nancy ont donc une approche pragmatique : l'étude phytosociologique se fait à deux échelles, elle commence par une compréhension écologique de la formation, pour être complétée par une étude floristique de l'association. Cette ouverture permet à Guinier d'assimiler les approches de l'écologie moderne et notamment de travaux américains fondateurs. Sans nommer McMillan, Cowles ou Clements, premiers écologues d'outreAtlantique, il prend pour exemple de dynamique des formations les dunes de l'Atlantique, à l'instar des travaux de Cowles sur les dunes du Michigan. Il intègre à son cours les notions de dynamique des associations, d'équilibre stable, d'évolution régressive ou progressive et même de climax, qualifiées d'essentielles pour orienter l'action des forestiers. Pratique, Guinier fournit à ses

${ }^{30}$ P. Acot et J.-M. Drouin, «L'introduction en France des idées de l'écologie scientifique américaine dans 1'entre-deux-guerres ", Symposium "Histoire de l'écologie et des sciences environnementales." XIX ${ }^{\mathrm{e}}$ Congrès international d'histoire des sciences de Saragosse, août 1993, à paraître. Je tiens à remercier les auteurs de leur bienveillance en me faisant état de leur travail sur cette question.

${ }^{31}$ P. Guinier, L'écologie forestière, enseigné par Philibert Guinier. Suivi de R. Rol, Le forestier devant la phytosociologie. Présenté par l'AFEF, ENGREF Nancy, 1995, 70 p. Il faut signaler, pour ne pas se méprendre que le titre donné à cet ouvrage est un effet d'éditeur, l'intitulé du cours de $\mathrm{P}$. Guinier était : cours de géographie botanique.

${ }^{32}$ Ibid., p. 55.

${ }^{33}$ ibid., p. 53. 
élèves les outils les plus récents d'analyse des formations végétales en leur présentant de façon détaillée les travaux récents de Raunkiaer de classification des paysages végétaux ${ }^{34}$. En 1953, R. Rol, dans la postface du cours de Guinier, rappelant les objectifs économiques de l'administration forestière, montre pourquoi ce dernier, dès 1903, comprit l'intérêt de l'approche écologique pour les forestiers.

Cet enseignement [la phytosociologie] devait rester pratique car ce que le forestier demande à l'étude des associations végétales, ce sont les indications précises sur l'écologie de la forêt et en vue de déterminer le traitement le plus rationnel, eu égard aux considérations économiques dont il ne peut se désintéresser. Cette remarque permet de comprendre pourquoi l'application intégrale aux forêts des théories phytosociologiques de l'école dite Züricho-montpellieraine, dont Braun-Blanquet est le chef incontesté et auxquelles se sont ralliés la majorité des phytosociologues, offre des difficultés réelles et ne répond pas toujours au but souhaitable. (P. Guinier, 1932) ${ }^{35}$

L'Ecole forestière de Nancy, dans ses besoins scientifiques pour satisfaire les demandes sociales de production de bois, intègre donc l'écologie à son enseignement dès l'entre-deux-guerres. Le travail des forestiers produit en retour des expériences qui nourrissent la réflexion des écologues. L'écologie scientifique passe ainsi, en s'alliant aux objectifs d'orthopédie forestière de l'Administration des Eaux et Forêts, du rang de savoir scientifique à celui de savoir pratique. Ceci explique que le vocable "écologie" soit encore peu usité dans la pratique discursive des forestiers qui le considèrent plus, pendant cette période, comme une pratique de bon sens que comme une science. Dans l'univers rationaliste et scientiste de l'école de Nancy, on lui préfère les mots "floristique" et "phytosociologie", plus intimement rattachés aux sciences de la vie et mieux admis dans le petit monde très sérieux de la botanique systématique française. Il n'en reste pas moins, comme le montre le cours de Guinier, que derrière ces termes, on trouve déjà les paradigmes écologiques de l'équilibre et du global, de la gestion de l'environnement et de sa fragilité.

Pour les forêts tropicales humides, cette position est encore plus marquée. Avant les travaux de botanistes et de biogéographes comme A. Aubréville et R. Schnell, dans les années cinquante, qui sur le terrain de l'Afrique équatoriale arrivent aux mêmes conclusions, P. Guinier met en exergue la pertinence de la notion de formation pour appréhender ce type de groupements végétaux.

\footnotetext{
${ }^{34}$ P. Guinier. ibid., p. 53 et 54. Cf. Raunkiaer, The life-forms of plants and statistical geography. Oxford, Clarendon Press, 1934. cité par J.-M. Drouin, L'écologie et son histoire...op.cit., p. 89. Signalons que les principales idées de Raunkiaer sont énoncées dès 1905.

${ }^{35}$ Ibid., p. 66.
} 
Dans les régions intertropicales, il est encore plus important de décrire les types de forêts et de connaître leur évolution. (P. Guinier, 1932)

Ceci est déjà vrai pour notre pays, où cependant la flore est relativement pauvre et fort bien connue, mais la notion de formation s'impose inéluctablement aux forestiers des pays tropicaux où la flore est d'une richesse extrême et où les études systématiques sont loin d'être terminées. (P. Guinier, 1932) ${ }^{37}$

Cet enseignement expliquerait que les administrateurs forestiers en Indochine aient été perméables aux principes de dynamique successionnelle de l'écologie américaine. R. Ducamp, dès 1912, recommande pour régénérer la forêt d'observer les cycles de formation de la végétation et devance en quelque sorte, par des observations empiriques, la problématique de l'écologue américain $\mathrm{F}$. Clements.

Pour refaire la forêt rémunératrice là où elle n'est plus, il faut recréer progressivement l'ambiance propice qui lui est chère, il faut repasser par ce que l'on a coutume d'appeler la brousse, en un mot il faut interdire de façon absolue le feu d'abord, le pâturage ensuite et remplacer la coupe de n'importe quelle herbe ou fougère par la coupe méthodique faite d'après les règles que fixe la sylviculture.(...) Ceci fait comprendre que ce serait pure folie de vouloir créer d'un seul coup des forêts ne renfermant qu'une seule essence ou un nombre très réduit (exception faite pour les peuplements de pins de filao et peut être de quelques dipterocarpacées), que ce serait folie de vouloir créer d'emblée des forêts à peuplement pur, constituées par une seule essence, alors que cette essence ne forme jamais en forêt de peuplement absolument pur ; les essences qui composent la forêt se sont remplacées, substituées suivant des lois à peine soupçonnées dans les pays mêmes où la science forestière est la plus avancée et où l'on dépense sans compter pour poursuivre toutes les expériences qui permettront d'énoncer ces lois de succession des essences sur un même sol, les lois de répartition, d'association, celles qui expliquent que telles essences sont grégaires, d'autres disséminées, d'autres exclusives. (R. Ducamp, 1912) ${ }^{38}$

La formule de R. Ducamp «observer la nature pour en accélérer son cuvre », va peu à peu triompher des projets utopiques de recomposition totale des forêts et ceci semble-t-il grâce à l'introduction d'une problématique écologique dans la gestion des forêts. Les observations vont alors montrer que la forêt tropicale humide se régénère d'elle-même, après un cycle d'une centaine d'années. Cependant, L. Lavauden, conservateur des Eaux et Forêts, ne croit pas à la possibilité d'une régénération complète des forêts, qu'elles soient tropicales ou tempérées ${ }^{39}$. Pour lui, seul se remet en place un subclimax (Landes de

\footnotetext{
${ }^{36}$ Ibid., p. 60.

${ }^{37}$ Ibid., p. 64.

${ }^{38}$ R. Ducamp, cité par H. Guibier, Caractères généraux des bois d'Indochine. op. cit., p. 188.

${ }^{39}$ L. Lavauden, Le problème forestier colonial. Paris, Nancy, Strasbourg, Berger-Levrault, 1931,
} p. 10 . 

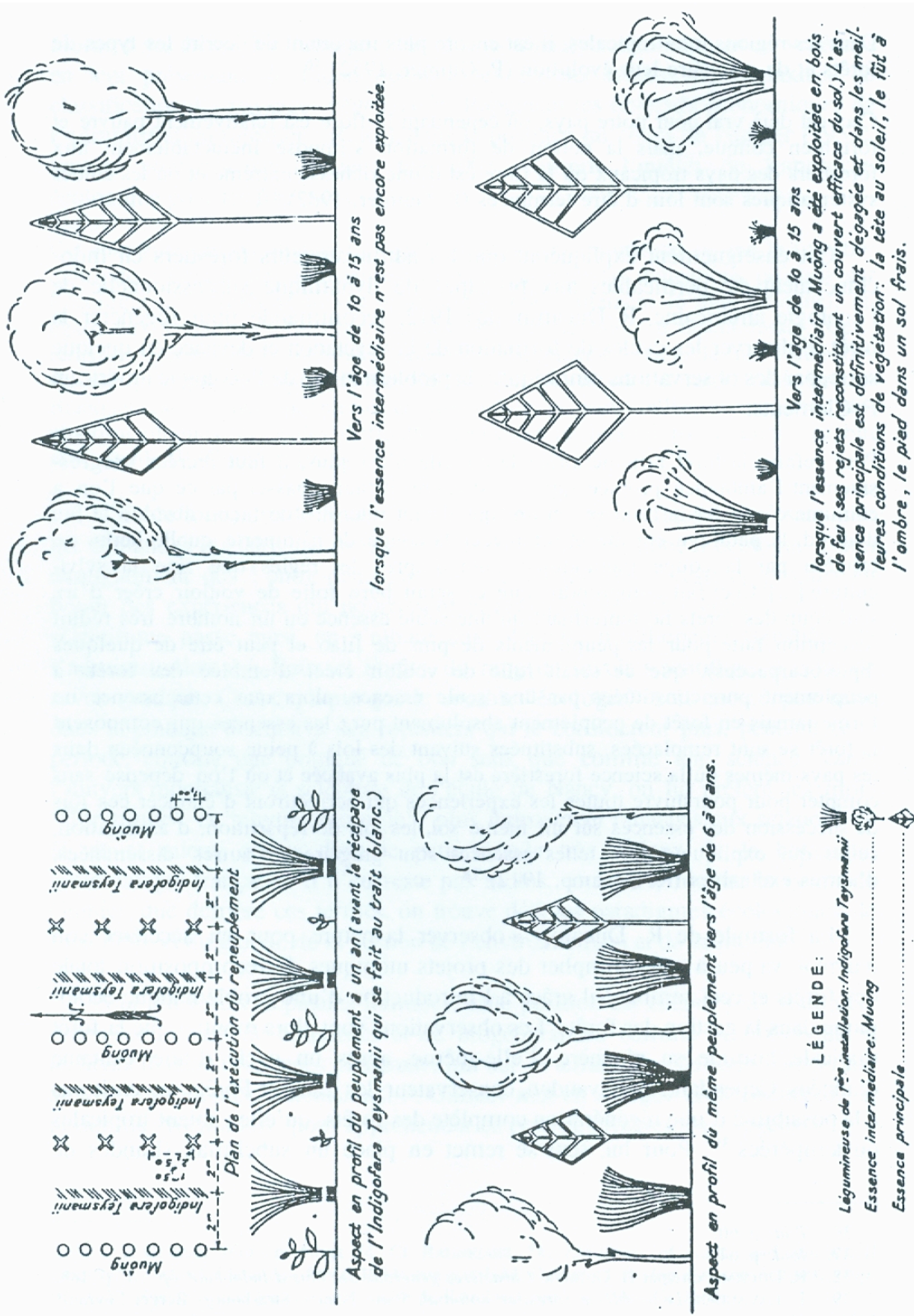
Calolune, Landes d'ajoncs, garrigues à lavande ou romarin, etc.). Ce stade de régénération avec un état stabilisé de subclimax est encore plus vrai pour la forêt tropicale humide. La richesse de la forêt primaire ne se reconstitue jamais, l'administration forestière, par un travail de balivage, doit viser l'enrichissement de la forêt secondaire. Le service forestier mit au point plusieurs méthodes d'enrichissement ; celle de taillis sous futaie fut la plus recherchée, car la plus adaptée aux besoins du marché local. Le taillis sous futaie concilie l'intérêt à long terme d'enrichissement en essences de bois dur et l'intérêt à court terme d'exploitation du bois de feu. Ce type d'aménagement a permis selon P. Maurand d'éviter en Cochinchine une crise du bois de feu. Au contraire, toujours selon lui, à Phnom-Penh l'absence de traitement a entraîné la multiplication des transports pour trouver du bois de feu et la multiplication de son prix par deux par rapport à Saigon $^{40}$. Le reboisement, deuxième grande œuvre d'aménagement forestier, concerne essentiellement en Indochine deux essences, le Filao pour les dunes de sable dont le rôle hydrologique est capital pour le maintien des dunes du centre de l'Annam, mais qui est aussi très rentable commercialement, et les Pins pour une exploitation en gemmage. Les techniques de plantation que rapporte $\mathrm{P}$. Maurand sont variées :

- Défricher à blanc estoc, consiste en un déboisement à blanc avec brûlage des rémanents pour reboiser en pins, mais cette technique ne peut se pratiquer qu'à proximité des pépinières car il faut pallier pendant 20 ans les problèmes du sol mis à nu.

- Les reboisements peuvent également se faire avec conservation d'un couvert naturel. Plusieurs essais montrent qu'un couvert épais et bas n'est pas efficace. Un couvert très relevé et léger, c'est-à-dire la conservation lors du défrichement du seul étage supérieur, permet le développement de jeunes essences.

- Le repeuplement avec concours d'une essence intermédiaire de remplissage, après une coupe à blanc estoc sans brûlage des rémanents est la dernière expérimentation du service forestier d'Indochine. On plante l'essence recherchée avec une essence intermédiaire, le plus souvent le Muông, mais cette technique ne fut jamais tout à fait satisfaisante.

- Le repeuplement dit de "méthode classique» avec le concours d'une légumineuse de première installation et d'une essence intermédiaire de remplissage est le plus utilisé en forêt dense. La technique est efficace mais nécessite quatre-vingts jours de travail et en terrain argilo-siliceux seulement.

La station expérimentale de Trang-Bom sert d'exemple à $\mathrm{P}$. Maurand pour décrire ces différentes techniques de repeuplement. Les schémas ci-contre illustrent la difficulté que les services forestiers eurent pour mettre au point des techniques de replantation assurant une exploitation commerciale facile, tout en s'adaptant aux conditions de compétition du climat chaud et humide.

\footnotetext{
${ }^{40}$ P. Maurand, L'Indochine forestière. op. cit., p. 13.
} 
Ces travaux confirment l'utilisation des concepts de dynamique successionnelle de la végétation par les services forestiers d'Indochine. Quand disparaît l'idée, très vivace après la première guerre mondiale, d'une France autosuffisante en bois grâce aux marchés de ses colonies, ces principes se concrétisent par des effets réels dans le choix des aménagements forestiers. L'utopie cède la place au réalisme et, avec $\mathrm{H}$. Guibier, chef du service forestier d'Indochine dans la dernière moitié des années vingt, l'idée nihiliste de créer à l'endroit des forêts tropicales humides des peuplements homogène disparaît.

Une des principales préoccupations fut, dès le début, d'enrichir les forêts en sujets d'essences classées; et cela par un balivage aussi parfait que possible, complété par des semis directs et des plantations de jeunes sujets élevés en pépinières, installés près des postes forestiers européens et indigènes, parfois en forêt même (...). Tous les essais entrepris amènent à cette conclusion qu'il est absolument inutile de tenter une expérience de semis, semis direct, à la volée, ni aucune expérience de mise en place de plants élevés en pépinière, si l'on ne peut pas suivre et protéger les semis et les jeunes plants jusqu'au moment ou ils sont assez grands et robustes pour ne plus craindre les plantes voisines $(\mathrm{H}$. Guibier, 1926) $)^{41}$.

Comme on le voit ici, et malheureusement pour la connaissance des forêts denses humides, ces concepts écologiques qui aux États-Unis stimulèrent des sensibilités conservationnistes, ne furent utilisés en Indochine, combinés aux principes de police de la Nature, qu'à des fins aménagistes et commerciales, sans qu'ils ne développent de recherches plus désintéressées sur le milieu lui-même. Seule la lutte contre le rẫy va se faire au nom de la protection de l'environnement global, c'est ce que nous allons observer pour finir.

\section{LE RẪY ET LES PEUPLES DES MONTAGNES D'INDO-CHINE}

La France, pendant la seconde moitié du $\mathrm{XIX}^{\mathrm{e}}$ siècle, a connu sur les déboisements un débat houleux qui opposait le pastoralisme pyrénéen et alpin aux ingénieurs des Ponts et Chaussées ainsi qu'aux forestiers de l'école de Nancy ${ }^{42}$. Ces derniers, dans le scientisme qui caractérisait l'époque, avaient imposé la triple vision d'un déboisement catastrophique du point de vue hydrologique, économique, et social. Les grandes inondations de 1856 à 1859

${ }^{41}$ Ibid., p. 257.

42 J.-P. Métaillé, « De la géographie des forestiers à la géographie contre les forestiers. » in Autour de Vidal de la Blache, la formation de l'école française de géographie. CNRS édition, 1993, p. 101. 
puis celle de 1875 apportèrent de l'eau à leur moulin et l'exode rural, phénomène relativement nouveau, nourrit la thèse de l'épuisement économique et social des régions de montagne. Le tout fut attribué à l'incurable bêtise de "l'indigène" hostile à tout progrès d'un système agro-pastoral dispendieux. Ce fut le temps des grands reboisements de la Restauration et du second Empire, des Brémontier et Chambrelant, de l'extension du domaine réservé contre le mouton! Dans les colonies, ce discours est plaqué sans plus d'analyse. Les objectifs de conversion et de combat contre les usages populaires en forêt, furent projetés sur le terrain colonial et les populations d'Indo-Chine se retrouvèrent d'emblée accusées de détruire leurs forêts. L'agriculture était désignée comme l'ennemi de la forêt, l'agriculteur, particulièrement dans les "pays au bois", par son état de sauvagerie directement lié à sa vie forestière, ruinait la forêt sans en être conscient ; il appartenait donc à l'administration de le protéger contre lui-même. Ces affirmations générales quoique souvent péremptoires trouvèrent peu de contradicteurs en France, malgré la présence du lobby pastoral puissant dans sa représentation politique ${ }^{43}$. Dans les colonies, le discours fut peut-être encore plus radical et les opposants encore plus rares, les populations accusées n'ayant pas droit de cité. On le retrouve à tous les niveaux, Gouverneurs généraux, forestiers, exploitants et mêmes géographes dénoncent à l'unisson l'agriculture de forêt (permanente ou pas) comme la principale raison d'appauvrissement, pis, de destruction, "des bois du domaine colonial".

En brossant un rapide tableau de la lutte des forestiers coloniaux contre le rẫy, culture itinérante sur brûlis, il ressort que c'est dans cette bataille que l'écologie scientifique est la plus invoquée dans l'argumentation du service forestier d'Indochine. A. Bertin dans l'introduction d'un recueil de synthèse sur la réglementation forestière dans les colonies françaises, après avoir fait un récapitulatif de l'importance des espaces forestiers dans les diverses colonies, accuse les populations locales "d'anéantir" les forêts. L'Indochine est particulièrement visée, les fortes densités de peuplement y sont perçues comme une lourde menace pour la forêt. "(...) les 20 millions d'habitants de notre Empire asiatique ont déjà anéanti de grands massifs. »(A. Bertin, 1924) ${ }^{44}$.

L'auteur poursuit son introduction en montrant l'importance des forêts sur la climatologie et son influence sur les sols : "L'influence des masses sylvestres est considérable sur la climatologie et la fertilité d'un pays. (...) les forêts sont en

\footnotetext{
${ }^{43}$ Ibid. p. 102. C'est particulièrement par l'intermédiaire des assemblées, et notamment le Parlement, que le lobby pastoral exercera son influence, mais il n'élaborera quasiment pas de discours scientifique. L'auteur signale quelques exemples de contre-discours, comme celui de Doumenjon. L'école française de géographie voulut rester en dehors de ce débat, en se démarquant de tout travail aménagiste. Même l'école de Grenoble et les travaux de R. Blanchard éludèrent la controverse.

${ }^{44}$ A. Bertin, Régimes forestiers dans les colonies françaises. op. cit., p. 4.
} 
réalité de grands réservoirs d'eau qui se comportent comme des éponges (...) L'absorption d'eau de pluie par le sol est de $6 \%$ en terrain nu, contre $61 \%$ en terrain boisé. » (A. Bertin, 1924) ${ }^{45}$.

Lavauden explique quelques années plus tard l'importance des régions boisées sur le régime des pluies, qui reçoivent 20 à $25 \%$ fois plus de pluie que le même terrain mis à nu. Même si les proportions sont discutées, une connaissance écologique apparaît de plus en plus clairement dans les années 30 pour montrer qu'il y a corrélation entre couverture végétale et le microclimat ambiant. Pour lui, le rẫy est l'unique vecteur de destruction de la forêt, disculpant au passage l'exploitation coloniale de toute destruction. «Le feu est le principal ou même l'unique auteur. » (L. Lavauden, 1931) ${ }^{46}$

Au Tonkin, H. Guibier confirme en 1938 que l'exploitation commerciale des bois de la colonie du début du siècle est trop faible pour user les forêts ; en 1910 le volume de tous les bois exploités dans cette colonie était de $43.575 \mathrm{~m} 3$ et en 1929 de $268.700 \mathrm{~m} 3$ sans grande variation sur les années trente ; les grands responsables des destructions des forêts du Tonkin sont donc les peuples montagnards. En 1943, P. Maurand estime à 10 millions d'ha la quantité de forêts détruites par an par le feu ou le pacage.

On relève systématiquement deux catégories de conséquences écologiques, pour les auteurs, dans la pratique du rẫy. La première concerne l'érosion. Les inondations et tous les problèmes hydrologiques sont perçus comme la conséquence directe des rẫy ; l'érosion des sols finit par rendre infertiles les vallées, lieu de la riziculture irriguée, par dépôt d'alluvions grossières, elle est responsable du nomadisme de l'agriculture de montagne car le sol, s'épuisant, ne permet pas l'établissement d'une agriculture permanente ; on passe ainsi de proche en proche à des considérations écologiques au sens large d'équilibre social.

En Annam sous l'action des incendies, multipliés par le système agricole des montagnards, la forêt a beaucoup perdu de sa compacité ; les pluies violentes des moussons ont permis une rapide progression des torrents, les basses rivières se chargent de plus en plus d'alluvions grossières et infertiles qui envahissent les petites plaines. Ici, il semble qu'il faille donner aux habitants de la plaine un droit de regard sur les forêts de la montagne. (P. Deffontaines, 1933) ${ }^{47}$

Le rẫy risque donc à terme de plonger l'Indo-Chine des montagnes, comme celle des vallées et des deltas, dans un bouleversement de son organisation économique et de son équilibre social. La responsabilité en revient aux peuples

\footnotetext{
45 ibid., p. 5 .

${ }^{46}$ L. Lavauden; Le problème forestier colonial. op. cit., p. 31.

${ }^{47}$ P. Deffontaines, L'homme et la forêt. op. cit., p. 172.
} 
montagnards et à leur agriculture pauvre et appauvrissante, et c'est au nom d'un équilibre global que P. Deffontaines préconise un droit d'ingérence (écologique) des peuples de la plaine sur ceux des montagnes.

Il existe pourtant des observations qui infirment cette image sans nuance des effets écologiques du rẫy sur l'environnement. Elles sont souvent le résultat de connaissances vernaculaires qui viennent contredire les lois de la science forestière européenne. On reconnaît par exemple l'utilité du rẫy dans le renouvellement des associations. Avec beaucoup de méfiance et en utilisant le conditionnel, les administrateurs forestiers reprennent les affirmations de "l'indigène" quand leurs connaissances scientifiques sont prises en défaut : "Le passage du feu pendant la saison sèche serait une sorte d'assainissement du sol, ce qui permet aux semis de s'installer et de croître rapidement et serrés, et serait même capable d'empêcher la mort des peuplements adultes. " (H. Guibier, $1926)^{48}$

Le même auteur rapporte que dès le début de la colonisation les incendies avaient été totalement interdits entre 1865 et 1867, dans les plaines de Biên Hoa en Cochinchine. Or selon le témoignage de A. Henry, chef de bataillon d'Infanterie de Marine, des invasions de sauterelles survinrent l'année suivante.

Mais au printemps de 1868, des nuées épouvantables de sauterelles s'abattirent sur les récoltes de Đồng-Nai, elles dévorèrent jusqu'au Tranh des toitures. Naturellement les indigènes attribuèrent cette invasion de sauterelles aux mesures qui avaient été prises pour empêcher les incendies des plaines de Tranh. Avaient-ils raison ? Nous ne saurions nous prononcer là-dessus, mais en tout cas, ils avaient les apparences pour eux, et les ont encore, car depuis cette époque on s'est relâché dans la surveillance stricte exercée pendant ces trois années précitées, et les sauterelles ne sont pas reparues. Que devons-nous conclure de ceci ? (H. Guibier 1938) ${ }^{49}$

Ce témoignage recèle une grande richesse quant aux rapports des coloniaux avec le milieu qu'ils découvraient et leurs contacts avec les populations. Ici, on ne peut que s'amuser du procédé de Guibier pour remettre en cause les conclusions indigènes, certes logiques mais quelque peu scolastiques. L'observation, pourtant longue, ne fait donc pas preuve, selon lui. Il reste dubitatif face à cette expérience qui va à l'encontre des enseignements forestiers. Pour nous, ceci montre qu'intellectuellement, dès le début du siècle, la réflexion scientifique pouvait remettre en cause les principes de causalité les plus simplistes quand ceux-ci paraissaient quelques peu baroques. Or, dans bien des cas, quand l'observation allait dans le sens de l'administration forestière, c'est le contraire qui se produisait, on avançait par exemple que le rẫy était responsable des inondations sans mettre en doute la certitude d'une observation pourtant aussi

\footnotetext{
${ }^{48}$ H. Guibier, Caractères généraux de la forêt indochinoise. op. cit., p. 264.

${ }^{49}$ H. Guibier, La forêt d'Indochine. op. cit., p. 59.
} 
peu vérifiable que le rapport absence de rẫy / invasion de sauterelles. Ceci révèle également que les forestiers n'étaient pas totalement ignorants des savoirs vernaculaires sur la forêt, qu'ils étaient même probablement quotidiennement confrontés à eux, mais ne pouvaient les envisager qu'avec la condescendance et la circonspection qui plaçaient la connaissance européenne au-dessus, au rang de science malgré le même degré d'empirisme. Tout était dans la formulation, le péremptoire asseyant la certitude, le processus d'inscription universalisant le savoir.

La seconde catégorie de conséquences constitue, nous semble-t-il, le fond de la logique de condamnation de cette pratique. Le rẫy appauvrit de façon irrémédiable la richesse en essences de bois dur, c'est à dire commerciales, des forêts de la Colonie: "En Indochine, après le rẫy, les essences forestières inférieures reprennent seules possession des cultures abandonnées.» (P. Deffontaines, 1933) $)^{50}$

Les observations phylogéniques de R. Ducamp en 1912 sur les cycles de reconstitution de la forêt, les expérimentations de H. Guibier sur les dynamiques successionnelles ont donné au service forestier la conscience de la plus ou moins grande richesse commerciale des climax forestiers. La forêt primaire, climax climacique, est riche en essences de bois dur, la forêt secondaire, climax biotique, est considérablement appauvrie dans sa variété floristique et particulièrement en essence de bois d'œuvre ou de première catégorie. C'est le rẫy, là encore, qui est rendu responsable de cet pauvreté de la forêt secondaire: "Il lui manquera toujours [la forêt secondaire] toutes les essences à graines lourdes qui ont été détruites par le feu. » (L. Lavauden, 1931) ${ }^{51}$

A propos des forêts du Tonkin où les forêts primaires n'existent plus, dès l'entre-deux-guerres d'après la plupart des auteurs, que sur quelques rares collines calcaires, le principal grief qui est fait au rẫy n'est pas tant la diminution de la richesse floristique de ces forêts primaires que la disparition des essences commerciales.

On ne trouve ni de vastes peuplements de conifères du sud Annam, ni la forêt avec prédominance de dipterocarpacées (...) Elles ont perdu presque tout leur caractère primitif ; les indigènes se livrant depuis des siècles à leur dévastation (...) dans les plaines, on ne trouve plus que la forêt mutilée, appauvrie en bonnes essences, (...) forêts clairières constituées par une petite brousse rabougrie fréquemment incendiée, ou, plus souvent encore, par des landes peuplées de chétifs arbustes, ou des savanes herbeuses recouvrant des surfaces immenses sur lesquelles s'étendit autrefois la grande forêt primaire. (H. Guibier, 1926) ${ }^{52}$

\footnotetext{
${ }^{50}$ Ibid., p. 43.

${ }^{51}$ L. Lavauden, Le problème forestier colonial. op. cit., p. 6.

${ }^{52}$ H. Guibier, Caractères généraux... op. cit., p. 272.
} 
En filigrane, on comprend que l'émotion suscitée par la destruction de la forêt primaire chez Guibier n'est pas l'appauvrissement de la richesse floristique ou la rupture d'un écosystème mais bien la disparition des essences à forte plus-value détruites par l'essartage. Dans ces conditions, l'argument écologique contre le rẫy est une justification fallacieuse qui vise à masquer le but commercial de la lutte contre cette pratique.

Face à ce constat, c'est l'urgence qui est décrétée ! Les méthodes de lutte contre le rẫy se concentrent dans la trilogie punir, surveiller, éduquer.

Le rẫy a subi des interdictions absolues dès le début de la colonisation, mais ces tentatives se révéleront impossibles à réaliser dans la pratique. En 1914, l'article 55 de l'arrêté du 27 mars, interdit toujours le rẫy, mais le service forestier d'Indochine reconnaît la nécessité d'autoriser la pratique dans certaines régions où cette agriculture s'impose.

Qu'ils s'en moquent, Man, Thô, Méo, de la constitution juridique des terres sur lesquelles ils vivent par le rây, sans pouvoir vivre autrement! A leur place, vous en feriez tout autant. Il faut d'abord vivre. Le meilleur procédé est encore de laisser continuer les rây, mais en les localisant sur des zones de surface suffisante pour y pratiquer chaque année le rây sur l'étendue indispensable à la récolte. (H. Guibier, 1938)

Cette tolérance est donc de bon sens pour le service forestier, elle doit être une arme de l'administrateur pour éduquer les populations et mieux les contrôler. Les droits d'usage font l'objet d'un permis.

Les villages forestiers et les habitants de ces villages ont seuls droit d'usage, dans les forêts situées sur leur territoire, sauf dans la réserve. Néanmoins toute coupe de bois nécessite l'autorisation du service forestier. Le permis est gratuit moyennant cinq journées de prestation dont l'imposition restreint le commerce clandestin des ventes de bois d'usagers. (A. Bertin 1924) ${ }^{54}$

En d'autre temps on eut parlé de champart, la République avait un autre vocabulaire : permis gratuit ! Bertin estime ce contrôle formateur pour les indigènes à qui l'on peut ainsi révéler les miracles de la science forestière et le bien-fondé de la coupe méthodique. Quant aux villages situés au milieu d'une forêt mise en réserve, ils devaient également quelques journées de corvée.

Pour les villages englobés en entier ou en partie dans les réserves forestières, une superficie égale à 1 ha par feu sera délimitée par le service forestier pour l'exercice des droits d'usage (A. Bertin, 1924) ${ }^{55}$.

\footnotetext{
${ }^{53}$ H. Guibier, La forêt d'Indochine. op. cit., p. 32.

${ }^{54}$ A. Bertin, Régimes forestiers dans les colonies françaises. op. cit., p. 16.

${ }^{55}$ Ibid., p. 16.
} 
Les villages forestiers doivent en compensation des droits particuliers qui leur sont reconnus, fournir au service forestier des journées de prestation votées par les Conseils Provinciaux pour entretien des chemins et travaux d'amélioration divers en forêt. (A. Bertin, 1924) $)^{56}$

Le paternalisme du colonisateur, souvent dénoncé ultérieurement, était dans la réalité trop timoré pour que l'autorité française s'exercât et c'est bien d'une arrogance toute seigneuriale dont on usait pour administrer "l'indigène". Mais si nous soulignons ces deux passages sur les droits d'usage de la population, c'est avant tout pour illustrer à quel point les modes de vie locaux purent être ignorés par le colonisateur. Bertin nous rapporte que 1 ha de forêt par feu était octroyé pour ces droits. Cette proportion mérite d'être comparée à l'excellent travail de J. Boulbet sur les paysans des forêts de la région de Blao dans le Đồng Nai. Celui-ci constate en 1975, après des décennies passées en forêt, que l'agriculture sur brûlis, pour se maintenir sans que l'équilibre écologique soit rompu, ne peut être pratiquée que dans des zones où la densité au $\mathrm{km}^{2}$ est inférieure à cinq habitants ${ }^{57}$, soit cinq habitants pour 100 ha. Les villages d'essarteurs placés dans le domaine réservé disposaient donc d'un espace octroyé par l'administration deux cents fois inférieur au maintien de l'équilibre de leur économie. Le colonisateur faisait fi de la gestion séculaire du milieu forestier par les populations locales et pour ceci mettait en place un arsenal de sanctions. Dans les procédures de répression contre les délits forestiers, la peine la plus lourde pèse sur les personnes qui pratiquent le rẫy sans autorisation; elles encourent 1 à 3 mois de prison et 20 à $250 \mathrm{FF}$ d'amende à la charge de la communauté villageoise $^{58}$.

A la punition, toujours source de tensions sociales, le colonisateur préfère, chaque fois qu'elles sont possibles, la surveillance et l'éducation. Les voies de communication rompent l'isolement et permettent plus ou moins un contrôle du territoire. Elles sont surtout un vecteur privilégié pour intégrer les populations montagnardes aux circuits économiques de l'économie de marché, pour les sédentariser peu à peu et donc mieux les discipliner: "L'ouverture de nouvelles voies de pénétration, de communication créant des échanges modifiera peu à peu le mode de vie des populations montagnardes, les stabilisera progressivement. » (H. Guibier, 1938) ${ }^{59}$.

La transformation du rapport homme / nature, c'est-à-dire, la rupture voulue de l'écologie traditionnelle des peuples forestiers, fait donc partie intégrante du dispositif du contrôle colonial. Bonnes consciences altruistes, certitudes scientifiques et paternalisme sont au rendez-vous et la mystification de leur rôle

\footnotetext{
${ }^{56}$ Ibid., p. 23.

${ }^{57}$ J. Boulbet, Paysans de la forêt. Ecole Française d'Extrême Orient, 1975, p. 40.

${ }^{58}$ Décret du 11 juillet 1907, par arrêté du Gouverneur Général.

${ }^{59}$ H. Guibier, La forêt d'Indochine. op. cit., p. 34.
} 
évite aux forestiers de regarder l'ethnocide en marche. P. Maurand, en toute naïveté, pour lutter contre les rẫy, préconise une éducation en profondeur des populations.

(...) l'administration pour arriver à des résultats définitifs devra entreprendre une œuvre profonde d'éducation de la masse et aussi lui donner les moyens de remplacer ce que le feu avait d'avantageux pour elle : facilité de se déplacer en forêt et nourriture du bétail en saison sèche. (...) Ce sera le rôle du service forestier d'ouvrir les routes qui débloqueront les villages forestiers et deviendront des itinéraires obligatoires pour les voyageurs. (...) La population indigène voyant son standing de vie amélioré, en sera reconnaissante à l'administration et fera un effort pour éviter les incendies, sans se rendre compte tout d'abord du but poursuivi (P. Maurand 1943) ${ }^{60}$.

C'est même un travail de propagande qui doit être fait selon Guibier pour éduquer la jeunesse à la sauvegarde de la forêt, et il cite des expériences qui selon lui devraient être généralisées.

La directrice du collège Đồng-Khanh, à Huê, eut l'heureuse idée de demander à ses élèves des dessins de propagande où sont présentés, sous les couleurs du désespoir (pleurez, pleurez nos yeux !) le pays déboisé, les ravages des inondations, et sous les couleurs riantes et plus seyantes à leur jeunesse et à leur gentillesse, le pays reboisé rosissant, verdissant, florissant et prospérant. Propagande, Propagande ! (...) Dans les écoles de l'intérieur également, depuis deux ans au moins, dictées, compositions françaises et annamites sur les sujets forestiers, sur l'utilité de la forêt, sur ses bienfaits, sur l'obligation de la respecter, de la protéger, tout en profitant de ses dons (H. Guibier, $1938)^{61}$.

Cette initiative est en fait plus ancienne puisque des dictées traitant du rôle et de l'utilité des forêts avaient été rédigées par M. Roy, inspecteur adjoint des Eaux et Forêts dès 1906 pour les élèves des écoles de Cochinchine. Nous en rapportons, pour finir, un exemple parmi beaucoup d'autres.

Les forêts servent admirablement nos intérêts économiques par les matériaux qu'elles nous livrent, elles améliorent la moralité humaine par le sens esthétique qu'elles développent et la culture intellectuelle qu'elles stimulent (...) Le soin que prennent les peuples de leurs forêts est pour ainsi dire le thermomètre de leur prospérité et de leurs progrès. ${ }^{62}$

Ainsi, l'écologie, en mettant en évidence la corrélation entre déboisement, inondations et érosion, justifiait la dépossession du droit d'usage de la forêt des

\footnotetext{
${ }^{60}$ P. Maurand, L'Indochine forestière... op. cit., p. 35.

${ }^{61}$ H. Guibier, La forêt d'Indochine. op. cit., p. 89.

${ }^{62}$ Archives Nationales du Vietnam (Hanoi), Dir. de l'Agriculture, des Forêts et du Commerce de l'Indochine, Série N 9, Dossier n 662.
} 
peuples des montagnes au nom de la protection de l'environnement et de leurs propres intérêts.

\section{CONCLUSION : \\ L’ÉCOLOGIE COMME EUPHÉMISATION DE LA FORCE PUBLIQUE}

Si nous devions résumer le travail du service forestier dans la transformation des forêts d'Indochine, nous pourrions dire sans nuance que toute la logique consistait à exploiter la forêt dans les conditions commerciales les plus propices à dégager de gros bénéfices: "La sylviculture s'impose pour faire rendre au peuplement le maximum de produits utiles.» (A. Chevalier \& D. Normand, $1946)^{63}$

Pour ce faire, les administrateurs ont conçu des techniques d'enrichissement plus ou moins proches les unes des autres. Chacun dans une proportion plus ou moins grande projetait les modèles de la sylviculture européenne. Au cours des années cependant, et pour leur rendre justice, une évolution est perceptible dans la gestion des forêts d'Indochine et les concepts qui les ordonnent. Des considérations écologiques font leur apparition. D'abord vœux pieux, elles deviennent utilitaires dans la régulation hydrologique et la préservation des sols. Elles entrent de plus en plus dans les paramètres qui vont conduire l'action du service forestier. D'autre part, le modèle des forêts homogènes à l'européenne, bien que toujours très vivant à la fin de la période qui nous occupe, tend à être corrigé par l'expérience d'une sylviculture tropicale. Deux écologies sont pratiquées parallèlement par le service forestier. La première, écologie scientifique, dans sa problématique globalisante des écosystèmes, justifie la protection de la forêt contre les populations locales et plus particulièrement les peuples des montagnes qui sont les grands prédateurs du milieu forestier. La seconde, économie de la nature, montre que la «nature tropicale » a mis à la disposition de l'homme un capital dont "la très sage disposition » doit faire l'objet d'une «police de la nature» pour reprendre des expressions linnéennes. La sylviculture pratiquée dans le cadre colonial combine savamment ces deux épistémologies de l'écologie faisant du service forestier un gendarme et un organisateur de l'espace forestier en vue d'une exploitation rationnelle de ce capital. Le concept d'écosystème forestier est utilisé pour protéger la forêt des interventions humaines, inéluctablement destructrices dans le cas du rẫy et pour montrer la pauvreté des formations secondaires. En revanche, il s'efface lorsqu'il s'agit de décrire l'exploitation coloniale qui, elle, trouve sa justification écologique dans une orthopédie de la Nature. Ce va-et-vient entre deux

\footnotetext{
${ }^{63}$ A. Chevalier \& D. Normand, Forêts vierges et bois coloniaux. op. cit. p.109.
} 
démarches épistémologiques de l'écologie brouille les finalités économiques du service forestier qui n'apparaissent ainsi que les conséquences fortuites d'une approche scientifique. Les doxosophes forestiers «imposaient la croyance que leur fausse science était parfaitement indépendante des demandes sociales (coloniales) qu'elle ne satisfaisait si bien que parce qu'elle affirmait hautement son refus de les servir ${ }^{64}$.

Un quart de siècle plus tard, ce travail permettra malgré tout à $M$. Devèze d'affirmer le rôle bienfaiteur de l'administration forestière coloniale, mère de la protection forestière à travers le monde, permettant à l'homme blanc un droit de regard sur des espaces bientôt décolonisés.

Les débuts de la colonisation, malgré les défrichements et les exportations de bois vers l'Europe, n'avaient pas été trop dommageables. (...) L'Europe est la mère de la science forestière et de l'enseignement forestier. (...) Malgré le retard de certaines civilisations, pour lesquelles la forêt est encore un territoire de chasse et de cueillette, ou bien territoire de pâturage et de pacage, la forêt est essentiellement aujourd'hui source dans le monde de matière première. Le bois est une des principales richesses de l'humanité. (...) Aussi la forêt est-elle peu à peu, sur l'initiative de l'Europe l'objet de soins pressants sur les continents. L'ONU (sous la forme de la F.A.O.) s'intéresse à ce précieux capital. (M. Devèze, 1965$)^{65}$

La politique des services forestiers coloniaux est effectivement une première expérience de gestion du milieu tropical par des concepts environnementaux venus d'Europe qui se substituent aux savoirs vernaculaires. Elle préfigure le détournement de l'éthique globalitaire de l'écologie à des fins impérialistes, que le sommet de Rio en 1992 nomme le droit d'ingérence écologique. Ce que F. Sabelli dénonce comme étant l'esprit d'un nouveau colonialisme ${ }^{66}$, est bien plutôt l'aboutissement contemporain d'une longue construction historique qui lie l'écologie dans ses racines à l'universalisme hégémonique de l'occident moderne. La domination coloniale dans cette construction est une période capitale d'ajustement empirique des constructions scientifiques de l'Europe, qui assure définitivement l'écrasement des savoirs locaux en donnant à ces

\footnotetext{
${ }^{64}$ P. Bourdieu, "Le champ scientifique." Actes de la Recherche en sciences sociales, 1976. L'auteur ramène la construction du discours scientifique à la "réalité objective" de l'individu qui l'élabore en terme de stratégie personnelle. "Les doxosophes, savants de l'apparence, ne peuvent légitimer et la dépossession qu'ils opèrent par la construction arbitraire d'un savoir ésotérique inaccessible au profane et la délégation qu'ils demandent en s'arrogeant le monopole de certaines pratiques ou de la réflexion sur cette pratique, qu'à condition d'imposer la croyance que leur fausse science est parfaitement indépendante des demandes sociales qu'elle ne satisfait si bien que parce qu'elle affirme hautement son refus de les servir." p. 100.

${ }^{65}$ M. Devèze, Histoire des forêts. Paris PUF, 1965, p. 123.

${ }^{66}$ Sous la direction de F. Sabelli, Ecologie contre nature. Développement et politique d'ingérence. Paris PUF, 190 p. Cf. notammant F. Sabelli, "L'éthique globalitaire et l'esprit du nouveau colonialisme.” p. 13. et C. Raffestin "Les ingérences paradoxales de la pensée écologique." p. 41.
} 
conceptions universelles l'illusion de valeurs opératoires. C'est en exhumant les conditions objectives de l'émergence de ces sciences, en l'occurrence les impératifs de la mise en valeur coloniale, que l'on peut démontrer la supercherie d'une éthique globale de l'environnement.

Frédéric THOMAS 